\title{
Electronic transport through EuO spin-filter tunnel junctions
}

\author{
Nuttachai Jutong, ${ }^{1}$ Ivan Rungger, ${ }^{2}$ Cosima Schuster, ${ }^{1}$ Ulrich Eckern, ${ }^{1}$ Stefano Sanvito, ${ }^{2}$ and Udo Schwingenschlögl ${ }^{3}$ \\ ${ }^{1}$ Institut für Physik, Universität Augsburg, 86135 Augsburg, Germany \\ ${ }^{2}$ School of Physics and CRANN, Trinity College, Dublin 2, Ireland \\ ${ }^{3}$ KAUST, PSE Division, Thuwal 23955-6900, Kingdom of Saudi Arabia
}

(Received 9 July 2012; revised manuscript received 11 September 2012; published 12 November 2012)

\begin{abstract}
Epitaxial spin-filter tunnel junctions based on the ferromagnetic semiconductor europium monoxide (EuO) are investigated by means of density functional theory. In particular, we focus on the spin transport properties of $\mathrm{Cu}(100) / \mathrm{EuO}(100) / \mathrm{Cu}(100)$ junctions. The dependence of the transmission coefficient and the current-voltage curves on the interface spacing and EuO thickness is explained in terms of the EuO density of states and the complex band structure. Furthermore, we also discuss the relation between the spin transport properties and the $\mathrm{Cu}-\mathrm{EuO}$ interface geometry. The level alignment of the junction is sensitively affected by the interface spacing, since this determines the charge transfer between $\mathrm{EuO}$ and the $\mathrm{Cu}$ electrodes. Our calculations indicate that $\mathrm{EuO}$ epitaxially grown on $\mathrm{Cu}$ can act as a perfect spin filter, with a spin polarization of the current close to $100 \%$, and with both the Eu-5d conduction-band and the Eu- $4 f$ valence-band states contributing to the coherent transport. For epitaxial $\mathrm{EuO}$ on $\mathrm{Cu}$, a symmetry filtering is observed, with the $\Delta_{1}$ states dominating the transmission. This leads to a transport gap larger than the fundamental EuO band gap. Importantly, the high spin polarization of the current is preserved up to large bias voltages.
\end{abstract}

DOI: 10.1103/PhysRevB.86.205310

PACS number(s): 75.50.Pp, 72.25.Dc

\section{INTRODUCTION}

Magnetic tunnel junctions using $\mathrm{MgO}$ as a tunnel barrier generally display large tunneling magnetoresistance (TMR) and are now widely used in read heads for ultra-high-density hard-disk drives as well as in random access memory devices. ${ }^{1}$ Currently, a room-temperature TMR ratio as high as 500\% can be reached in junctions made of polycrystalline $\mathrm{Fe}$ (more precisely, $\mathrm{CoFeB}$ ) sandwiching a thin $\mathrm{MgO}$ insulating layer. ${ }^{2}$ Despite such huge TMR ratios, the $\mathrm{Fe} / \mathrm{MgO}$ system presents two limiting aspects for future applications. First, there is no flexibility on the materials side, which means that high TMR ratios are achieved only for the $\mathrm{Fe} / \mathrm{MgO}$ combination and only for a particular crystal orientation, i.e, (100). Furthermore, high crystallinity is needed as the electrodes themselves carry only a moderate spin polarization of the conduction electrons. Second, the TMR consistently decreases with increasing the applied voltage and/or the temperature, and so the operation of a practical device utilizing a magnetic tunnel junction is limited to low bias. ${ }^{3}$

The high $\mathrm{TMR}^{4,5}$ found in $\mathrm{Fe} / \mathrm{MgO}$-based junctions is caused by the symmetry filtering provided by the $\mathrm{MgO}$ barrier, together with the particular spin polarization of the Fe electrodes. Only electrons with $\Delta_{1}$ symmetry and with small transverse momentum contribute significantly to the current $^{6-12}$ through $\mathrm{MgO}$, and in $\mathrm{Fe}$ these are found only in the majority spins for electrons at the Fermi energy, so that the $\mathrm{Fe} / \mathrm{MgO}$ stack acts like a spin filter in the current flow. For energies around the Fermi energy, $E_{F}$, these are found only in the majority $\mathrm{Fe}$ states. As a consequence of this symmetry filtering, the decay rate of the conduction electron wave function into the $\mathrm{MgO}$ barrier of the majority spins is smaller than that of the minority ones. ${ }^{9}$ These decay rates are determined by the complex band structure (CBS) of the tunnel barrier. ${ }^{4,5}$ A crucial feature of the symmetry spin-filtering effect is that TMR ratios larger than $1000 \%$ can be achieved, ${ }^{5}$ despite the fact that the spin polarization of the electrodes is only around $65 \%$ (for $\mathrm{CoFeB}$ ). We note that the Julliere model, ${ }^{10,13}$ which predicts a much smaller TMR, fails in the present context.

Another possible strategy for obtaining a spin-filter effect, and thus very large TMR ratios, is that of using a ferromagnetic insulator as a spacer between the metallic electrodes. In this case, the energy barrier has a different height for majority and minority spins, which leads to the suppression of the current for one of the two spin species for thick-enough junctions. The tunnel current density is proportional to the energy-dependent transmission coefficient through the barrier, $T(E)$, which itself depends exponentially on the barrier height $\Phi$ and the barrier thickness $t$. This can be written as $T(E) \propto \exp [-2 \kappa(\Phi, E) t]$, with $\kappa(\Phi, E)=\sqrt{2 m_{e}(\Phi-E)} / \hbar$, and with $m_{e}$ being the electron mass. Clearly, if the barrier height is different for the different spin species, then the current polarizations will increase exponentially with the insulating layer thickness, leading to full spin polarization for thick barriers. ${ }^{3,12}$ Devices constructed with this principle are called spin-filter tunnel junctions (SFTJs). The quest for manufacturing SFTJs then reduces to that of finding suitable ferromagnetic semiconductors.

The europium chalcogenides $\mathrm{EuS}$, EuSe, and $\mathrm{EuO}$ all have a rocksalt structure and are all ferromagnetic insulators. Among these, EuO presents the largest conduction-band exchange splitting, $\sim 0.54 \mathrm{eV}$, below the material's Curie temperature of $69 \mathrm{~K} \cdot{ }^{14-16} \mathrm{In} \mathrm{EuO}$, the divalent Eu ions possess a large local moment originating from the half-filled $4 f$ band $\left(\mu_{\mathrm{Eu}}=7 \mu_{B}\right)$. An energy gap of $1.0 \mathrm{eV}$ separates the half-filled majority Eu- $4 f$ band from the $\mathrm{Eu}-5 d$ conduction band. ${ }^{3}$ SFTJs based on polycrystalline EuO in the form of a $\mathrm{metal} / \mathrm{EuO} / \mathrm{metal}$ heterojunction have been studied in several recent experiments. ${ }^{16-22}$ However, the spin transport properties of crystalline epitaxial EuO junctions have not been studied theoretically so far. The purpose of this paper is to provide such theoretical insight. In particular, we present ab initio results for the electronic structure and the electron transmission through 
EuO barriers sandwiched between $\mathrm{Cu}$ electrodes oriented along the [001] direction.

Our paper is organized as follows. We start our discussion by presenting the methods used and the structure of the device investigated. Then, in the following Sec. III, we present the electronic structure of $\mathrm{EuO}$ and its complex band structure along the [001] direction. This determines the spin-dependent decay rates and thus the spin-filter efficiency. In Sec. IV, we discuss the transmission coefficient at zero bias, while in Sec. V, the dependence of the spin transport on the EuO thickness is analyzed and related to the complex band structure. The current-voltage $(I-V)$ characteristics are discussed in the following section, where we find that in contrast to $\mathrm{Fe} / \mathrm{MgO}$ junctions, the polarization of the current is not reduced by the applied bias. Finally, we conclude.

\section{METHODS}

The $\mathrm{EuO}$ and $\mathrm{Cu}$ electronic structures are calculated by using the $a b$ initio density functional theory (DFT) code SIESTA. $^{23}$ Since the local density approximation (LDA) is not sufficient to give an accurate description of the $\mathrm{EuO}$ density of states (DOS), we correct for on-site Coulomb repulsion with an LDA $+U$ treatment. $^{24,25}$ Following Ref. 26, the exchange constant $J$ and the on-site orbital potential $U$ for the Eu- $4 f$ orbitals are set, respectively, to $J_{f}=0.77 \mathrm{eV}$ and $U_{f}=8.3 \mathrm{eV}$, while for the O-2p orbitals, we use $J_{p}=$ $1.2 \mathrm{eV}$ and $U_{p}=4.6 \mathrm{eV}$. Troullier-Martins norm-conversing relativistic pseudopotentials are used for $\mathrm{Cu}, \mathrm{Eu}$, and $\mathrm{O}$. The wave functions are expanded over a double- $\zeta$ plus polarization (DZP) basis set, except for the Eu- $4 f$ states for which we use single $\zeta$. An equivalent real-space mesh cutoff of $600 \mathrm{Ry}$ is used together with an electronic temperature of $69 \mathrm{~K}$. We sample the Brillouin zone (BZ) in the plane perpendicular to the transport direction over a $7 \times 7 k$ point uniform mesh.

Spin transport is studied by using the SMEAGOL electronic transport code, ${ }^{27-29}$ which combines DFT with the nonequilibrium Green's function (NEGF) technique. SMEAGOL uses the Hamiltonian matrix provided by SIESTA to calculate the charge density, so that the same pseudopotentials and exchange and correlation function can be used for both the electronic structure and the transport. The spin current at each bias voltage is calculated by energy integration of the spin-dependent transmission coefficient $T^{\sigma}$,

$$
I^{\sigma}=\frac{e}{h} \int d E T^{\sigma}(E, V)\left[f\left(E-\frac{e V}{2}\right)-f\left(E+\frac{e V}{2}\right)\right],
$$

where $\sigma$ is the spin index ( $\sigma=\uparrow, \downarrow), f$ is the Fermi function, $V$ is the applied bias voltage, and $e$ is the electron charge. The total transmission coefficient is obtained by integrating the $k$-dependent transmission $T^{\sigma}\left(E, V, k_{x}, k_{y}\right)$ over the twodimensional BZ perpendicular to the transport direction,

$$
T^{\sigma}(E, V)=\frac{1}{\Omega} \int d k_{x} d k_{y} T^{\sigma}\left(E, V, k_{x}, k_{y}\right),
$$

where $\Omega$ is the area of the BZ. We denote $T(E)=T(E, V=0)$ as the zero-bias transmission coefficient. In our calculations, we assume the lattice structure to be periodic in the $x-y$ plane, and we keep the transport direction along the $z$ axis.

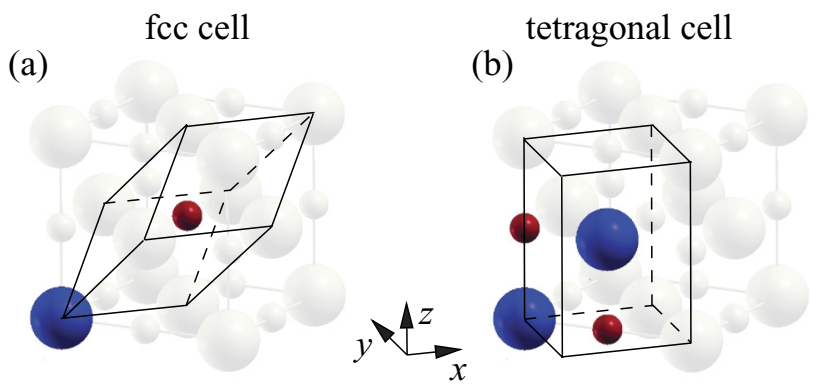

FIG. 1. (Color online) Lattice structure of EuO constructed with (a) the primitive fcc unit cell and (b) the tetragonal unit cell. The shaded gray atoms indicate the full cubic cell. Atom code: large spheres $($ blue $)=\mathrm{Eu}$, small spheres $($ red $)=\mathrm{O}$.

While $7 \times 7 k$ points are enough to accurately converge the charge density, the presence of resonances in $T^{\sigma}\left(E, V, k_{x}, k_{y}\right)$ requires the much larger mesh of $100 \times 100$ to evaluate the transmission coefficient both for zero and finite bias. The $I-V$ characteristics are calculated non-self-consistently by evaluating the transmission coefficient over an effective bias-dependent Hamiltonian matrix, which in turn is obtained by adding a rigid shift to the zero-bias Hamiltonian matrix elements of the electrodes and a linear potential across the insulating barrier. This is a good approximation to the selfconsistent potential drop for tunnel junctions, ${ }^{6}$ which appears essentially like that of a standard parallel-plate capacitor.

EuO crystallizes in the rocksalt structure with a lattice constant of $a_{\mathrm{EuO}}=5.144 \AA$. The primitive face-centered cubic (fcc) unit cell containing one Eu and one $\mathrm{O}$ atom is shown in Fig. 1(a). Since we consider transport along the [001] direction, we have to use, as a basic building block for the $\mathrm{EuO}$ spacer, a cell with size and orientation different from that of the primitive one. The smallest possible cell thus has tetragonal symmetry, and contains two Eu and two $\mathrm{O}$ atoms [see Fig. 1(b)]. By stacking multiples of such tetragonal cells along the $z$ direction, we can construct barriers of arbitrary thickness, where one cell contains two EuO monolayers (MLs).

The model spin-filter junction considered here consists of $n$ MLs of EuO sandwiched between nonmagnetic $\mathrm{Cu}$ electrodes, with $n$ being an integer. As electrode material, we consider fcc $\mathrm{Cu}$ oriented along the [001] direction (lattice constant 3.61 $\AA$ ), which is a material choice that has been adopted in several experiments. ${ }^{17-20}$ When the tetragonal $\mathrm{EuO}$ unit cell is used, the lattice constant of $\mathrm{Cu}$ can be matched with only a slight strain to the EuO lattice, since the dimensions along $x$ and $y$ are $a_{\mathrm{EuO}} / \sqrt{2}=3.63 \AA$. The junction setup is illustrated in Fig. 2. In this basic setup, both the $\mathrm{O}$ and $\mathrm{Eu}$ atoms are placed over the

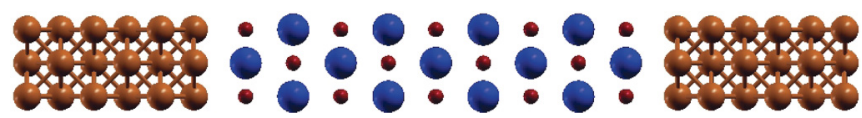

FIG. 2. (Color online) Supercell used for the spin-filter tunnel junction. This consists of six Cu MLs (left lead), nine Eu MLs (scattering region), and five $\mathrm{Cu}$ MLs (right lead). Atom code: large spheres (blue) $=\mathrm{Eu}$, medium spheres (brown) $=\mathrm{Cu}$, small spheres $($ red $)=\mathrm{O}$. 
hollow sites of the $\mathrm{Cu}$ surface. We also perform calculations for EuO shifted in the $x-y$ plane. However, the transport and spin-filter properties are not sensitively dependent on such a shift. The equilibrium interface distance along the $z$ direction, $d$, between $\mathrm{Cu}$ and $\mathrm{EuO}$ is found to be $d=2.8 \AA$. This value is plausible when comparing with the interface between $\mathrm{Au}$ and $\mathrm{MgO}$, which is found to be $2.5 \AA .^{30,31}$ Note that we do not consider possible oxidation at the $\mathrm{Cu} / \mathrm{EuO}$ interface, or the formation of secondary $\mathrm{EuO}$ phases.

\section{EuO ELECTRONIC STRUCTURE}

Let us start our discussion by describing the electronic structure of EuO. The calculated $\mathrm{EuO}$ band structures for both the primitive fcc and the tetragonal unit cells are presented, respectively, in Figs. 3 and 5. The results agree rather well with previously published calculations. ${ }^{26,32}$ In order to compare the band structures for two cells, we use the same $k$-space path. Standard high-symmetry points are specified for the primitive fcc $\mathrm{BZ}$ and also for the primitive tetragonal $\mathrm{BZ}$ (where applicable). We note that due to band folding for the tetragonal cell, we find twice the number of bands than for the primitive fcc one. The $X$ point [located at $\left(\pi / \mathrm{a}_{\mathrm{EuO}}, 0,0\right)$ ] and the $X^{\prime}$ point [located at $\left.\left(0,0, \pi / \mathrm{a}_{\mathrm{EuO}}\right)\right]$ are equivalent in the fcc BZ. However, for the tetragonal cell, they are independent since the $X^{\prime}$ point is equivalent to $\Gamma$ in the tetragonal cell. There is a band gap of about $1.0 \mathrm{eV}$ for majority spins (Fig. 3), whereas the gap increases to about $3.5 \mathrm{eV}$ for the minority spins. For the primitive fcc cell (Fig. 3), we find an indirect gap, where the top of the valence band is at $\Gamma$ and the bottom of the conduction band is at the $X^{\prime}$ point. This gap becomes direct when the primitive tetragonal cell is used due to band folding (Fig. 5). Note that the tetragonal cell is the relevant one for the transport, so that $\mathrm{EuO}$ along the [001] direction behaves as a direct-gap ferromagnetic semiconductor.

We first consider the electronic band structure of the majority spins, for which the energy spectrum consists of three parts: the conduction bands, the top valence bands, and the lower valence bands. The top valence bands are found in the range between -1.6 and $-0.5 \mathrm{eV}$ (we set the zero energy approximately at the middle of the gap). It is clearly seen from Fig. 4, where we show the projected DOS (PDOS) on different orbitals, that the main character of the top valence band is given by the Eu- $4 f$ states. While many of the Eu- $4 f$ bands show very little dispersion, indicating strong localization, in
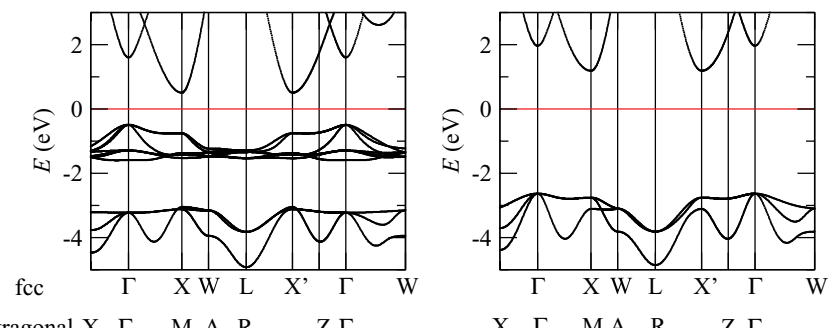

tetragonal $\mathrm{X} \quad \Gamma \quad \mathrm{M} \mathrm{A} \quad \mathrm{R} \quad \mathrm{Z} \Gamma$

FIG. 3. (Color online) LDA $+U$ band structure of $\mathrm{EuO}$ for the primitive fcc unit cell [see Fig. 1(a)]. Left panel: majority-spin bands; right panel: minority-spin bands. The band structure shows an indirect band gap from $\Gamma$ to $X$ of about $1.0 \mathrm{eV}$. Note that the line $\Gamma-X^{\prime}$ contains the same information as the line $\Gamma-X$.

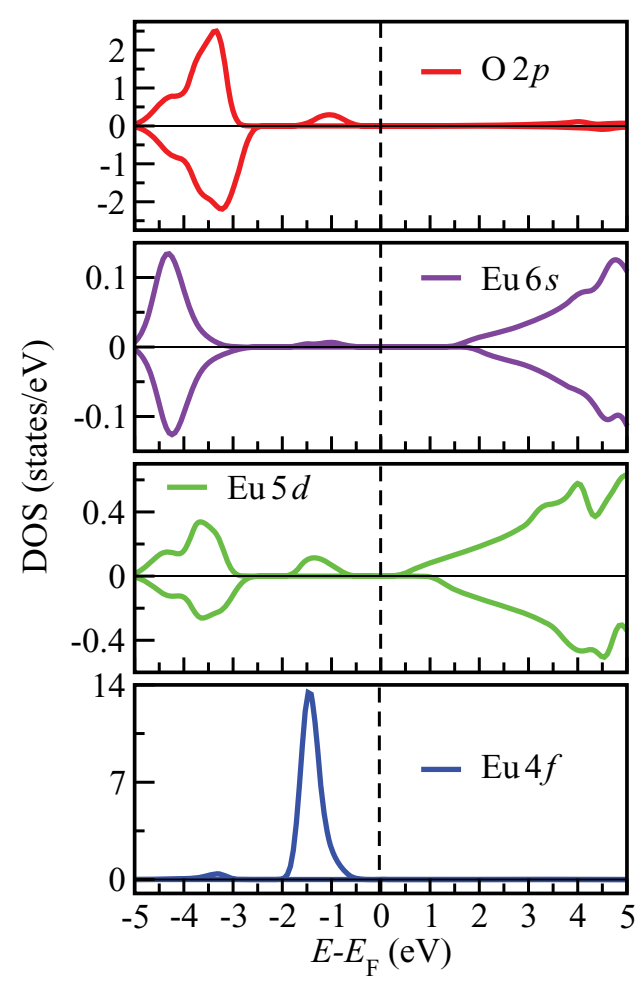

FIG. 4. (Color online) EuO density of states projected over the following atomic orbitals: $\mathrm{O}-2 p, \mathrm{Eu}-6 s, \mathrm{Eu}-5 d$, and $\mathrm{Eu}-4 f$.

$\mathrm{EuO}$ the $\mathrm{Eu}-4 f / \mathrm{O}-2 p$ mixing leads to a significant dispersion around the $\Gamma$ point. This is an indication of delocalized states. We note that at the top of the valence band, Eu- $4 f$ and O- $2 p$ contribute approximately equally to the density of states. In addition, the hybridization leads to a large contribution to the intersite coupling of kinetic processes. ${ }^{33}$ We therefore expect such delocalized states to contribute significantly to the transport through a EuO barrier. Below the top valence bands, the bottom valence bands are observed in the energy range from -4.9 to $-3.0 \mathrm{eV}$. It is seen from Fig. 4 that the bottom valence bands are dominated by $\mathrm{O}-2 p$ states. The bandwidth of the $\mathrm{O}-2 p$ states $(1.9 \mathrm{eV})$ is not much larger than that of the Eu- $4 f$ states $(1.0 \mathrm{eV})$, which indicates a similar wave-function delocalization. The energy gap between the top valence and bottom conduction bands is about $1.0 \mathrm{eV}$. The
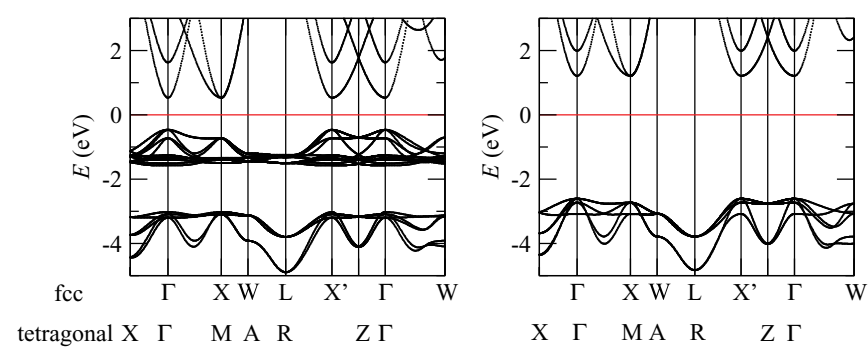

FIG. 5. (Color online) $\mathrm{LDA}+U$ band structure of $\mathrm{EuO}$ for the primitive tetragonal unit cell [see Fig. 1(b)], with majority-spin bands in the left panel and minority-spin bands in the right panel. Due to the doubled size unit cell, compared to the primitive fcc one, a band down-folding makes the $\Gamma$ point and the fcc $X^{\prime}$ point equivalent. This leads to a direct gap at $\Gamma$. 
lower conduction bands are dominated by Eu-5d states. Above $1.6 \mathrm{eV}$, we find also contributions from the Eu- $6 s$ orbitals. We note that the DOS at the lower end of the conduction bands is very small, which is due to a high dispersion.

Next we consider the electronic band structure of the minority spins. The empty Eu-4 $f$ states start at around $11.1 \mathrm{eV}$, so that they are not expected to affect the transport properties. As for the majority states, the conduction bands are dominated by Eu-5d and Eu- $6 s$ orbitals, while the valence bands are dominated by O- $2 p$. The spin-filter character of EuO is due to a spin splitting of the bands, which leads to a difference in the band gaps for the majority and minority spins. The splitting between the majority- and minority-spin conduction bands is calculated to be about $0.6 \mathrm{eV}$, in agreement with previous theoretical studies ${ }^{26,32-36}$ and with recent experiments using three-dimensional (3D) angle-resolved photoemission spectroscopy. ${ }^{37,38}$ The Eu-6s states show a spin splitting similar to the Eu-5d states, namely, $0.4 \mathrm{eV}$.

We now analyze the symmetries of the different bands. Symmetries are important for the transport through epitaxial tunnel junctions ${ }^{4,39}$ since, at any given energy, only evanescent states in the insulating barrier with matching symmetry with propagating Bloch states in the electrodes can contribute to the conductance. Since the EuO gap in the tetragonal cell is at $\Gamma$, the transport will be dominated by states close to $k_{x}=k_{y}=0$, denoted as $\Gamma_{2 \mathrm{D}}$, for which the barrier height is smallest (the coordinate system is defined in Fig. 1). In the energy range comprised between -1.8 and $1.6 \mathrm{eV}$ around $E_{F}$, the $\mathrm{Cu}$ electrodes have only states of $\Delta_{1}$ symmetry with respect to the $z$ axis. For a cubic space group, the $\Delta_{1}$ symmetry transforms as a linear combination of 1 ( $s$ orbitals), $z\left(p_{z}\right.$ orbitals), $2 z^{2}-x^{2}-y^{2}\left(d_{z^{2}}\right.$ orbitals), and $z\left(2 z^{2}-3 x^{2}-3 y^{2}\right)$ ( $f_{z^{3}}$ orbitals). Above $1.6 \mathrm{eV}$, the $\mathrm{Cu}$ states have $\Delta_{5}$ symmetry. The $\Delta_{5}$ symmetry transforms as a linear combination of $x\left(p_{x}\right.$ orbitals), $y$ ( $p_{y}$ orbitals), $x z$ ( $d_{x z}$ orbitals), and $y z$ ( $d_{y z}$ orbitals). At energies below $-1.8 \mathrm{eV}$, we find the $\mathrm{Cu}-3 d$ orbitals, so that there are also states with different symmetries. For a transport measurement up to about $2 \mathrm{~V}$, we therefore expect the states with $\Delta_{1}$ symmetry to determine the transport properties.

In order to investigate in more detail the propagating and evanescent states in the EuO barrier, we calculate the complex band structure (Fig. 6) at $\Gamma_{2 \mathrm{D}}$ (Refs. 4, 39, and 40) and analyze the symmetries of the evanescent states. Real wave vectors $\left(\operatorname{Im}\left[k_{z}\right]=0\right)$ represent propagating states and complex wave vectors; $\operatorname{Im}\left[k_{z}\right] \neq 0$ represent evanescent states since their wave functions decay as $\exp \left(-\operatorname{Im}\left[k_{z}\right] \cdot z\right)$ across the barrier. For the majority spins, the top of the valence band is threefold degenerate, with one state with $\Delta_{1}$ symmetry (Eu-4 $f_{z^{3}}$ and O- $2 p_{z}$ orbitals) and two states with $\Delta_{5}$ symmetry. Since the state with $\Delta_{1}$ symmetry has a lower effective mass than those with $\Delta_{5}$ symmetry, the corresponding $\Delta_{1}$ evanescent states have smaller $\operatorname{Im}\left[k_{z}\right]$, and therefore a slower decay. The bottom of the conduction band is given by a state with $\Delta_{2}$ symmetry (Eu-5 $d_{x^{2}-y^{2}}$ orbital). At $1.6 \mathrm{eV}$, we find the Eu- $4 s$ states, which have $\Delta_{1}$ symmetry. Therefore $\operatorname{Im}\left[k_{z}\right]$ for the $\Delta_{1}$ band forms a semicircle between the Eu- $4 f_{z^{3}}$ states at the top of the valence band and the Eu- $4 s$ states at $1.6 \mathrm{eV}$.

Consequently, we expect the tunneling transmission to be dominated by the $\Delta_{1}$ states in this energy range. The Eu-5d propagating states with $\Delta_{2}$ symmetry cannot couple to the

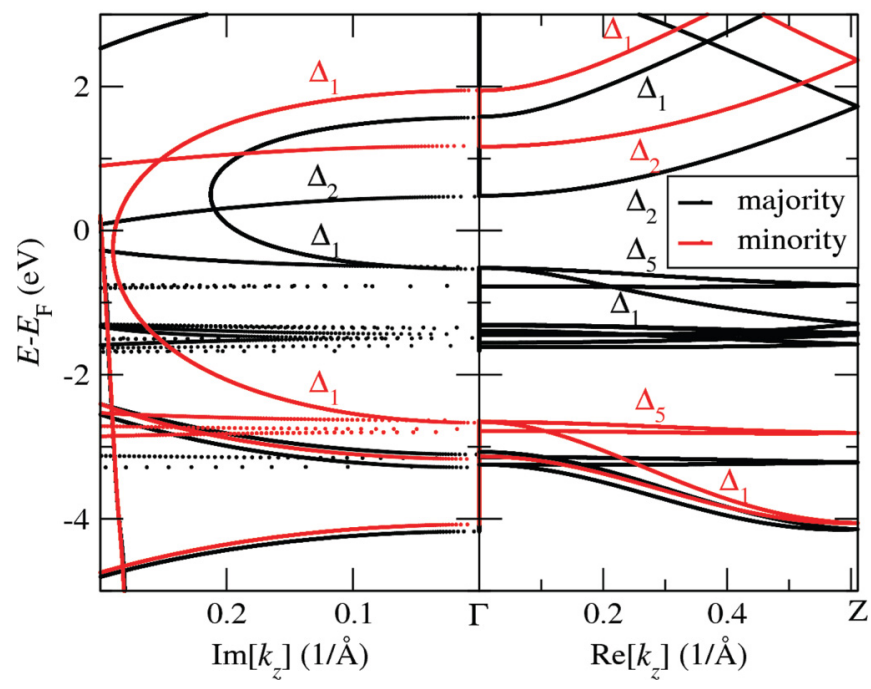

FIG. 6. (Color online) Complex band structure of EuO in its tetragonal cell [see Fig. 1(b)], where the symmetries are indicated for the bands relevant to our transport setup. Red lines = minority spins, black lines = majority spins.

$\Delta_{1} \mathrm{Cu}$ states, and therefore are not expected to contribute significantly to the transmission. For $k_{x}$ and $k_{y}$ different from zero, the Eu-5d states are no longer fully orthogonal to the $\mathrm{Cu}-4 s$ states. However, since the gap increases with increasing $k_{x}$ and $k_{y}$, the barrier height for such states is larger. Hence their contributions to the total transmission are smaller. Overall, one expects a rather weak coupling to the $\mathrm{Cu}-4 s$ states and a low transmission for states at the bottom of the EuO conduction band.

In the minority-spins bands, there are also three degenerate states at the top of the valence band: one with $\Delta_{1}$ symmetry $\left(\mathrm{O}-2 p_{z}\right.$ orbital) and two with $\Delta_{5}$ symmetry $\left(\mathrm{O}-2 p_{x}\right.$ and $\mathrm{O}-2 p_{y}$ orbitals). The $\Delta_{1}$ state again has lower effective mass and therefore a smaller decay for evanescent states. At the bottom of the conduction band, the symmetries are analogous to those of the majority spins. We therefore also expect the minority conductance to be dominated by the $\Delta_{1}$ symmetry. However, since the minority band gap is much larger than that of the majority spins, $\operatorname{Im}\left[k_{z}\right]$ is also significantly larger for $\Delta_{1}$ states in the gap. As a consequence, we expect $T^{\uparrow}$ to be substantially larger than $T^{\downarrow}$. This is usually attributed only to different energies of the bottom of the conduction band, ${ }^{3}$ whereas our complex band structure analysis indicates that the decay of the evanescent states has significant contributions also from states in the valence band, especially for the majority spins. We note that the Eu- $5 d \Delta_{2}$ states will contribute to the transport if different electrodes, possessing $\Delta_{2}$ symmetry states in the relevant energy range, are used.

\section{SPIN TRANSPORT PROPERTIES OF THE EUO JUNCTION AT ZERO BIAS}

In this section, we analyze the zero-bias transport properties of $\mathrm{Cu} / \mathrm{EuO} / \mathrm{Cu}$ junctions by taking as an example a stack containing nine MLs of EuO $(t=20.58 \AA)$. The energy-level alignment between the metal and the insulator is an important factor determining the transport properties of the junction. 


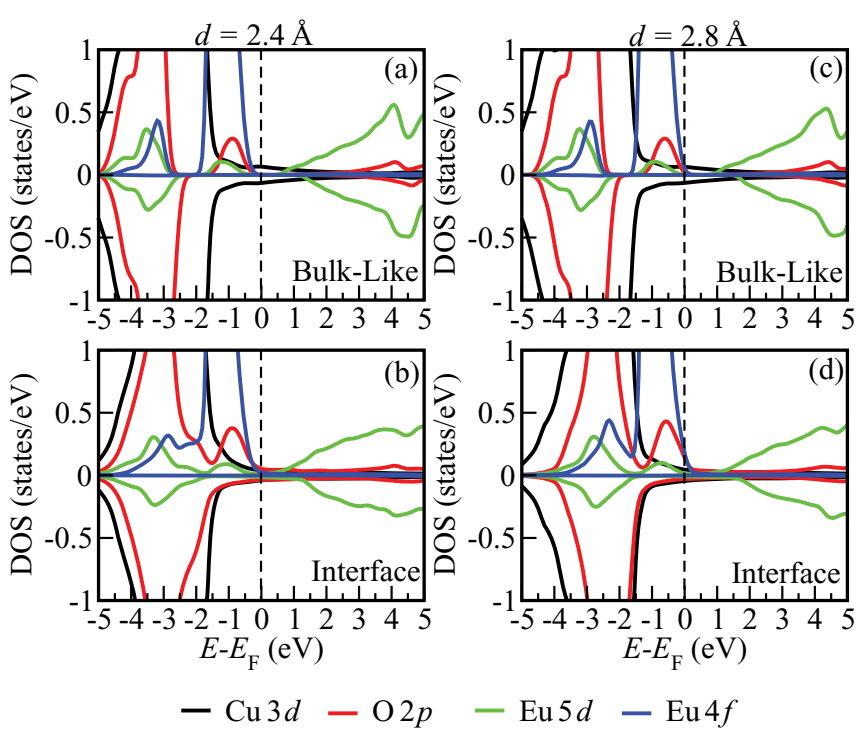

FIG. 7. (Color online) PDOS for $\mathrm{Cu}, \mathrm{O}$, and $\mathrm{Eu}$ for a junction comprising nine MLs EuO. The PDOS is calculated at (a) the center of the junction and (b) the interface for $d=2.4 \AA$, and at (c) the center of the junction and (d) the interface for $d=2.8 \AA$. Here, $d$ is the distance between the $\mathrm{Cu}$ and the $\mathrm{EuO}$ planes at the $\mathrm{EuO} / \mathrm{Cu}$ interface.

To a first approximation, one can estimate the alignment by comparing the work functions, $W$, of the two components. The work functions of $\mathrm{EuO}$ and $\mathrm{Cu}$ are calculated by using the Hartree electrostatic potential, $V_{H}$, as the reference potential. ${ }^{41}$ We define $W_{\mathrm{Cu}}$ as the difference between $E_{F}$ and the vacuum potential $V_{\text {vacuum }}$ of a $\mathrm{Cu}$ slab, while $W_{\mathrm{EuO}}$ is given by the difference between $V_{\text {vacuum }}$ and the energy of the valenceband top of a EuO slab, $V_{\mathrm{VB}}$. We calculate $W_{\mathrm{Cu}}=3.9 \mathrm{eV}$, which is somewhat lower than the theoretical values, ranging between 4.5 to $5.3 \mathrm{eV}^{42}$ and the experimental value of about $4.65 \mathrm{eV}^{43,44}$ Although the value of the calculated work function depends sensitively on the used parameters, we find that the transmission coefficient does not change significantly. The value for $\mathrm{EuO}$, i.e., $W_{\mathrm{EuO}}=1.8 \mathrm{eV}$, is in good agreement with the experimental value of $1.7 \mathrm{eV}$. ${ }^{45}$ Since $W_{\mathrm{EuO}}<W_{\mathrm{Cu}}$, electron transfer from $\mathrm{EuO}$ to $\mathrm{Cu}$ can be expected at the interface, leading to the pinning of the valence-band top of $\mathrm{EuO}$ to the $\mathrm{Cu} E_{F}$. This is indeed the case for the equilibrium distance $(d=2.8 \AA)$, where the Eu- $4 f$ states are located just below $E_{F}$ [see Figs. 7(c) and 7(d)].

In experiments investigating bias-dependent transport, the actual junctions usually contain polycrystalline $\mathrm{EuO}$, so that the interface between $\mathrm{Cu}$ and $\mathrm{EuO}$ is not well defined. ${ }^{22}$ In our calculations, in contrast, we assume a perfect epitaxial interface and do not explicitly consider the formation of defects, oxidation of $\mathrm{Cu}$, or the possible formation of $\mathrm{Eu}_{2} \mathrm{O}_{3}$ at the interface. ${ }^{17,21}$ The effect of such modifications of the interface on the electronic structure are manyfold, with the most important being that they usually lead to a different charge transfer and therefore to a different band alignment between $\mathrm{Cu}$ and $\mathrm{EuO}$. In practice, this means that (depending on the detailed structure of the interface, which is determined by the experimental conditions) $E_{F}$ can be placed at different positions across the EuO gap. In our calculations, we can tune the charge transfer and consequently the position of $E_{F}$ in the EuO gap by modifying the distance $d$ between $\mathrm{Cu}$ and $\mathrm{EuO}$ at the interface. We find that by decreasing the distance from the equilibrium one, i.e., increasing the coupling between $\mathrm{Cu}$ and $\mathrm{EuO}$, the $\mathrm{EuO}$ states shift to lower energies with respect to $E_{F}$. For $d=2.4 \AA, E_{F}$ is located approximately in the middle of the gap [see Figs. 7(a) and 7(b)], while for $d=2.2 \AA$, it is pinned at the EuO conduction-band minimum (not shown). Therefore, $d=2.2 \AA$ can be used to simulate the transport properties of $n$-type $\mathrm{EuO}$, as obtained for O-deficient barriers. ${ }^{46-48}$ In the remainder of this paper, we will present the transport properties for both $d=2.4 \AA$ and $d=2.8 \AA$, in order to illustrate the effect of a shift of $E_{F}$ induced by interface modifications. Importantly, we will demonstrate that for any position of $E_{F}, \mathrm{EuO}$ always shows excellent spin-filter characteristics up to high bias voltages. We note that if experimentally a perfect epitaxial junction can be realized, then the measurements should correspond to our results for the equilibrium distance $d=2.8 \AA$.

In Fig. 7, we compare the PDOS of atoms at the $\mathrm{Cu} / \mathrm{EuO}$ interface with the PDOS of atoms at a maximal distance from the interface (to be considered bulklike). While the two PDOSs are similar for most atomic orbitals, there is a significant difference for the O-2 $p$ states. At the interface, the O- $2 p$ states extend over a larger energy range as compared to bulklike atoms, indicating a coupling to the $\mathrm{Cu}$ substrate. In fact, for $d=2.4 \AA$, the broadening is more pronounced than for $d=2.8 \AA$, which is due to the larger coupling. The PDOS in the middle of the EuO layer is very similar to that of bulk EuO, indicating that in the middle of the junction, one recovers the bulk electronic structure of EuO. Although in Fig. 7 only the fully occupied $\mathrm{Cu}-3 d$ states are shown, we note that the $\mathrm{Cu}-4 s$ states determine the transport properties of the electrodes since they have an approximately constant PDOS in a large energy range around $E_{F}$. In Fig. 7, the $\mathrm{Cu}-4 s$ PDOS is not shown, since it is not visible on the chosen scale.

The zero-bias transmission coefficient $T(E)$ for different $d$ is shown in Fig. 8 [on a linear scale in Fig. 8(a) and on a logarithmic scale in Fig. 8(b)]. Due to the shift of the electronic states for different $d$ (see Fig. 7), $T(E)$ is shifted towards higher energies for $d=2.8 \AA$ as compared to $d=2.4 \AA$, while there is only a minor change in the height of the different transmission peaks. It can be seen that $T(E)$ has two small gaps for the majority spins and one large gap for the the minority ones. For the minority spins, the conduction occurs only through the O-2 $p$ states for energies below $-2.5 \mathrm{eV}$ and through the Eu-5d and Eu-6s states for energies above about $2 \mathrm{eV}$. For the majority spins, conduction occurs through the same states, and there is an additional high-transmission region between about -1.5 and $-0.5 \mathrm{eV}$, which is due to transport through the Eu- $4 f$ states. We note that at the bottom Eu-4 $f$ band edge at about $-1.5 \mathrm{eV}$, the transmission drops sharply by ten orders of magnitude, while at the upper band edge at around $-0.5 \mathrm{eV}$, the decrease is less pronounced and more gradual. This is consistent with the results from the complex band structure, which shows a sharp increase of the exponential decay $\operatorname{Im}\left[k_{z}\right]$ below the bottom band edge and a gradual increase above the upper band edge. It reflects the high localization of the Eu- $4 f$ states at the bottom band edge, in contrast to the top of the valence bands where the Eu- $4 f$ states 

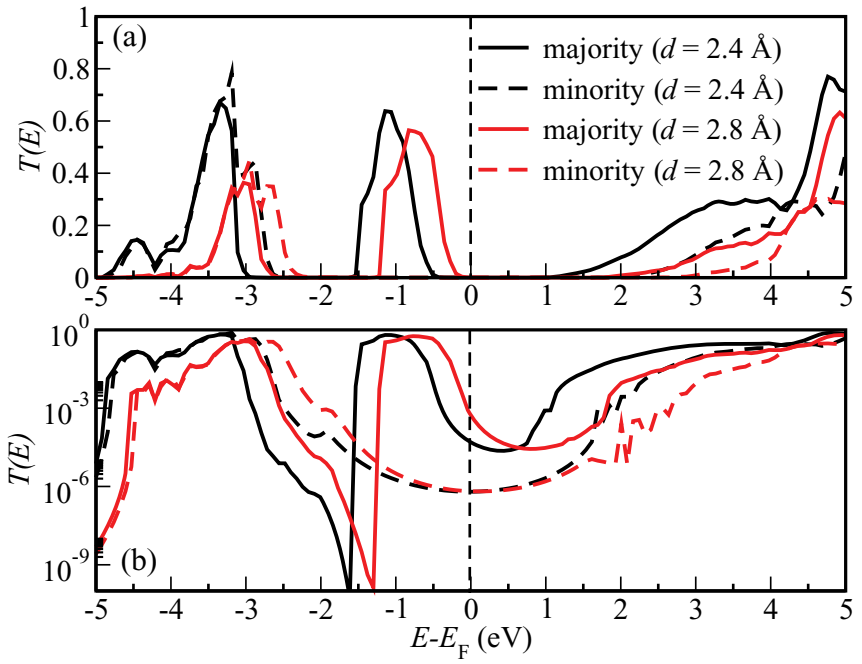

FIG. 8. (Color online) Transmission coefficient of a $\mathrm{Cu} / \mathrm{EuO} / \mathrm{Cu}$ junction formed by nine EuO MLs for $d=2.4 \AA$ and $d=2.8 \AA$, plotted on a (a) linear and a (b) logarithmic scale. Note that for $d=2.8 \AA, T(E)$ shifts to higher energy when compared to the case of $d=2.4 \AA$.

hybridize with the O-2 $p$ states and are rather delocalized. The Eu- $4 f$ states just below $E_{F}$ also contribute significantly to the conductance. This is consistent with the band structure, which shows that the Eu- $4 f$ states hybridize with the O- $2 p$ and are rather delocalized. The result of such hybridization is a large majority transmission in the energy range between $-1.5 \mathrm{eV}$ and $E_{F}$. Therefore, for all energies below $E_{F}$ (down to about $-1.5 \mathrm{eV}$ ), the transmission of the majority spins is much larger than that of the minority ones. For energies above $E_{F}$ (up to about $2 \mathrm{eV}$ ), it is also significantly larger. This is due to fact that the Eu- $5 d$ conduction-band minimum is located about $0.6 \mathrm{eV}$ lower in energy for the majority spins than for the minority ones (Fig. 6).

The overall result hence is that for any position of $E_{F}$ in the $\mathrm{EuO}$ gap, we expect a very high spin polarization of the current. This should persist up to high bias voltages (of the order of the energy for which the transmission of the majority spins is much larger than that of the minority ones). The spin-filtering efficiency at small applied bias is defined as $\left[T^{\uparrow}\left(E_{F}\right)-T^{\downarrow}\left(E_{F}\right)\right] / T\left(E_{F}\right)$. ${ }^{49}$ Since $T^{\uparrow}\left(E_{F}\right)$ is about two to three orders of magnitude larger than $T^{\downarrow}\left(E_{F}\right)$, we have a spin-filter efficiency close to $100 \%$, indicating that the EuO barrier is an almost perfect spin filter. We note that the extremely high efficiency is obtained for defect-free epitaxial junctions. Such value might be reduced by defects in the EuO barrier as well as for polycrystalline EuO. Nevertheless, one can expect that also in these cases, the spin-filter efficiency may remain high.

In order to investigate the dependence of the spin transport properties on the geometry of the $\mathrm{Cu} / \mathrm{EuO}$ interface, we calculate $T(E)$ for different positions of $\mathrm{EuO}$ with respect to $\mathrm{Cu}$. So far we have considered a setup in which $\mathrm{O}$ is placed on top of the hollow site of the $\mathrm{Cu}$ surface. We compare the results with the following three geometries: (1) O placed on top of $\mathrm{Cu}$, (2) $\mathrm{O}$ placed on the bridge site between two $\mathrm{Cu}$ atoms, and (3) $\mathrm{O}$ placed at an arbitrary low-symmetry site.
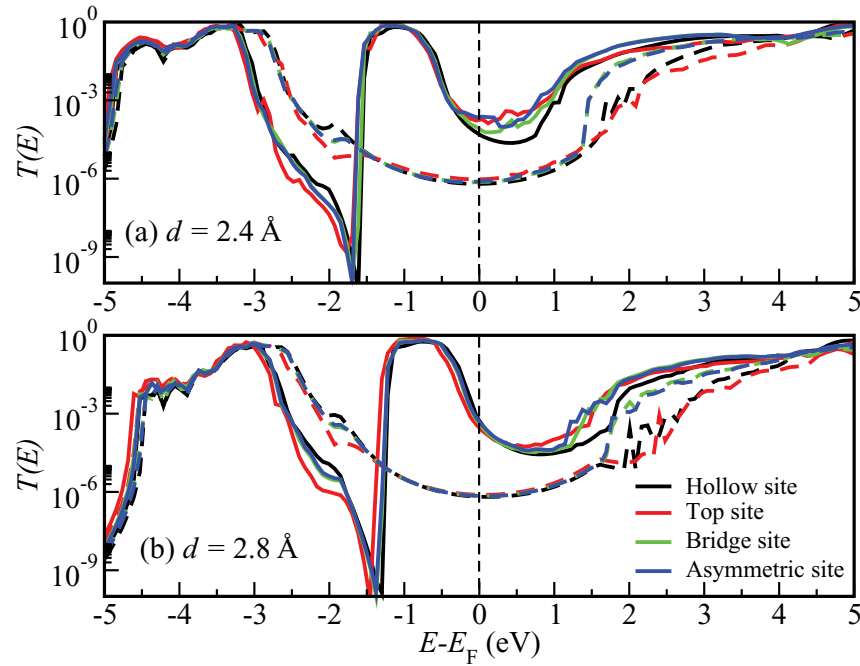

FIG. 9. (Color online) Transmission coefficient for a $\mathrm{Cu} / \mathrm{EuO} / \mathrm{Cu}$ junction with a nine MLs EuO barrier and with different interface geometries: (a) $d=2.4 \AA$ and (b) $d=2.8 \AA$. The solid lines represent the majority-spin transmission, whereas the dashed ones are for minority spins. Note that overall the transmission depends little on the lateral position of the $\mathrm{O}$ atoms with respect to the $\mathrm{Cu}$ surface.

An interlayer distance of $d=2.8 \AA$ minimizes the energy for the hollow site and is used also for the other configurations to ensure comparability of the transport results. In Fig. 9, we show $T(E)$ calculated for the different sites, for both $d=2.4 \AA$ and $d=2.8 \AA$. Overall, the changes in transmission corresponding to the different sites are rather small, a fact which indicates that the geometry of the $\mathrm{Cu} / \mathrm{EuO}$ surface does not significantly affect the band alignment and transport properties of the junction.

At the bottom of the conduction band, there are some quantitative differences in transmission between the different sites. This is due to the fact that shifting the $\mathrm{O}$ atom with respect to $\mathrm{Cu}$ alters the coupling between the $\mathrm{Cu}-4 s$ and Eu- $5 d$ states. As discussed in Sec. III, while the states on the top of the valence band couple well to the $\Delta_{1} \mathrm{Cu}$ band, the states at the bottom of the conduction band couple only slightly to these states due to a symmetry mismatch. This is also the reason for the fact that the transmission gap is somewhat larger than the $\mathrm{EuO}$ band gap, and for the slow increase of the transmission at the bottom of the conduction band with energy. For the bridge and low-symmetry sites, the symmetry mismatch is slightly reduced, resulting in a somewhat smaller transmission gap. Since the results for the different sites are very similar, we will only consider the hollow site in the remainder of the paper.

\section{THICKNESS DEPENDENCE OF THE CONDUCTANCE}

In this section, we analyze the effect of the EuO thickness $t$ on the spin transport properties of the $\mathrm{Cu} / \mathrm{EuO} / \mathrm{Cu}$ junction. The transmission spectra for different EuO thicknesses, and for both $d=2.4 \AA$ and $d=2.8 \AA$, are shown in Fig. 10. From the figure, it can be seen that the band alignment is not affected by the thickness, while the transmission in the gap decreases exponentially with it. Since for energies in the gap we have $T^{\sigma}(E) \propto \exp \left[-2 \kappa^{\sigma}(E) t\right]$, we can estimate $\kappa^{\sigma}$ from the change 


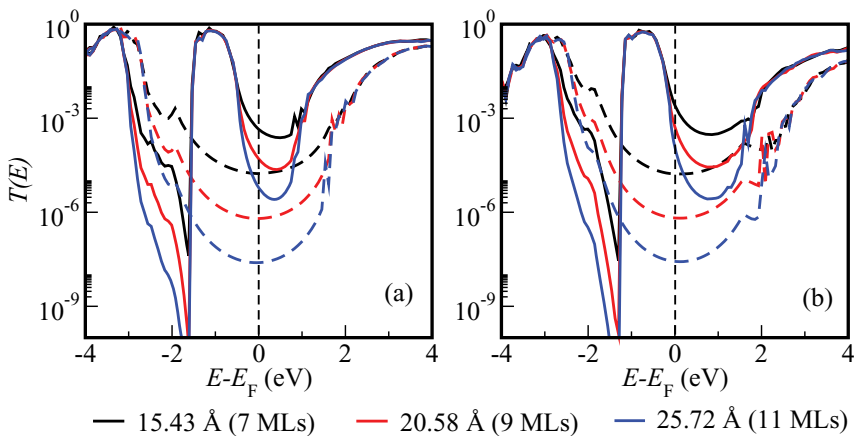

FIG. 10. (Color online) Thickness dependence of $T(E)$ at zero bias for (a) $d=2.4 \AA$ and (b) $d=2.8 \AA$. The solid and dashed lines refer to the majority and minority spins, respectively. At $d=$ $2.4 \AA$, the tunneling gap of the majority spins is found from -0.45 to $1.0 \mathrm{eV}$, while for the minority ones it extends from -2.5 to $1.6 \mathrm{eV}$. At $d=2.8 \AA$, the tunneling gaps of the majority and minority spins for all thicknesses range between -0.1 to $1.75 \mathrm{eV}$ and -2.3 to 2.5 $\mathrm{eV}$, respectively.

of $T^{\sigma}(E)$ with increasing $t$. Indeed, $\kappa^{\sigma}$ can be calculated from $T^{\sigma}$ evaluated at two different thicknesses $t_{1}$ and $t_{2}$ as

$$
\kappa^{\sigma}(E)=\frac{1}{2\left(t_{2}-t_{1}\right)} \ln \left[\frac{T_{1}^{\sigma}(E)}{T_{2}^{\sigma}(E)}\right],
$$

where $T_{1}^{\sigma}$ and $T_{2}^{\sigma}$ are the transmissions for $t_{1}$ and $t_{2}$, respectively.

In Fig. 11, we show the calculated $\kappa(E)$ (solid lines), evaluated for $t_{1}=20.58 \AA$ (nine EuO MLs) and $t_{2}=25.72 \AA$ (11 EuO MLs). For energies within $\pm 1.5 \mathrm{eV}$ around $E_{F}, \kappa^{\uparrow}$ is significantly smaller than $\kappa^{\downarrow}$. Therefore, increasing the EuO layer thickness leads to an enhancement of the ratio between the transmissions of the majority and minority spins, i.e., to an increase of the spin-filtering efficiency. For $d=2.4 \AA$, $\kappa^{\uparrow}$ is larger than zero in the range from -0.5 to $1.0 \mathrm{eV}$
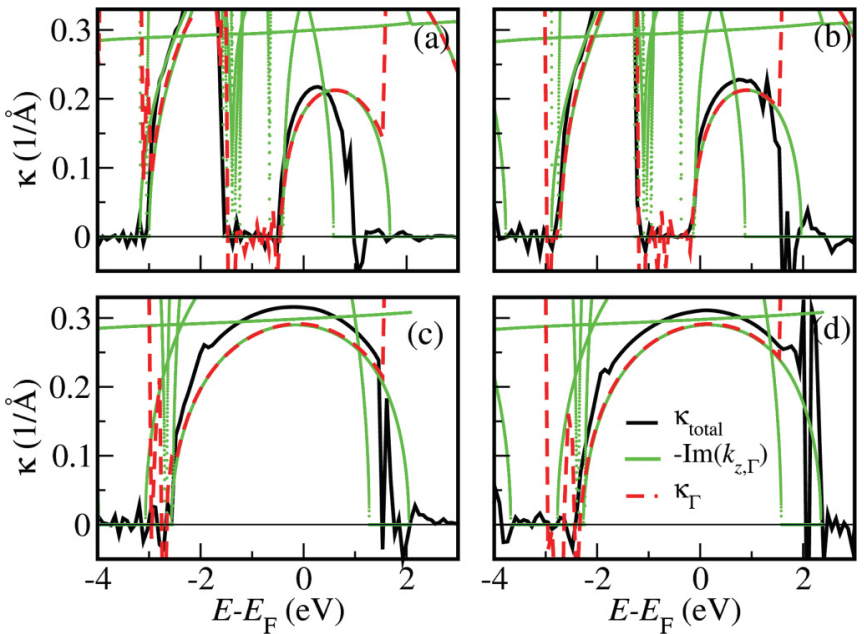

FIG. 11. (Color online) Damping coefficient $\kappa$ calculated from $T(E)$ for the (a),(b) majority and (c),(d) minority spins at $d=2.4 \AA$ and $d=2.8 \AA$. $\kappa$ is calculated from Eq. (3) by using the transmission coefficient calculated for nine and $11 \mathrm{EuO}$ MLs. The solid lines refer to the total transmissions and the dashed lines refer to the transmissions at $k_{x}=k_{y}=0$ only. The green lines show $\operatorname{Im}\left[k_{z}\right]$ from Fig. 6, which is also calculated for $k_{x}=k_{y}=0$. (resulting in a transmission gap of $1.5 \mathrm{eV}$ ), whereas $\kappa^{\downarrow}$ is larger than zero in the range from -2.6 to $1.6 \mathrm{eV}$ (resulting in a transmission gap of $4.2 \mathrm{eV}$ ). For $d=2.8 \AA, \kappa$ is shifted to higher energies, and the transmission gap amounts to about $1.7 \mathrm{eV}$ for the majority spins and $4.7 \mathrm{eV}$ for the minority ones. While $\kappa$ shows a parabolic behavior for energies close to the valence-band top, for the conduction-band minimum the behavior is less well defined. This is due to the symmetry mismatch between the $\mathrm{Cu} \Delta_{1}$ states and the conduction-band Eu-5d states; see Sec. IV. Nevertheless, the barrier height is about $0.6 \mathrm{eV}$ larger for the minority spins as compared to the majority ones for transmission through the conduction band. For the transmission through the valence band, the difference is even larger, since there are no filled minority-spin Eu- $4 f$ states.

In Fig. 11, we also present the $\kappa(E)$ obtained from the transmission only at the $\Gamma_{2 \mathrm{D}}$ point (dashed curve). Close to the valence band, the wave-function decay is similar to the previous case, which shows that $T(E)$ is dominated by contributions around $\Gamma_{2 \mathrm{D}}$. However, for energies close to the conduction band, $\kappa(E)$ at $\Gamma_{2 \mathrm{D}}$ is much larger than the total $\kappa(E)$, which shows that here the transport occurs mainly at $k$ points away from the $\mathrm{BZ}$ center. A comparison with the EuO complex bands (green curves; see Fig. 6 for a description of the symmetries) shows that at $\Gamma_{2 \mathrm{D}}, \kappa(E)$ follows approximately $\operatorname{Im}\left[k_{z}\right]$ for $\Delta_{1}$ states. It is clear that no transmission occurs through the conduction-band $\Delta_{2}$ states. For $k$ points away from $\Gamma_{2 \mathrm{D}}$, the $\mathrm{EuO} \Delta_{2}$ states can couple to the $\mathrm{Cu}$ states, which leads to a decrease of the total $\kappa(E)$ above the conduction-band minimum. For electrode materials with $\Delta_{2}$ states above $E_{F}$, we expect the total $\kappa(E)$ to follow the $\Delta_{2}$ complex bands at $\Gamma_{2 D}$ for energies below the conduction-band minimum.

The barrier heights are calculated from the damping coefficient shown in Fig. 11. The values of $\Phi_{\mathrm{CB}}^{\uparrow}$ and $\Phi_{\mathrm{CB}}^{\downarrow}$ are determined as the lowest energy at which $\kappa(E)$ crosses zero in the conduction band for the majority and minority spins, respectively. Similarly, $\Phi_{\mathrm{VB}}^{\uparrow}$ and $\Phi_{\mathrm{VB}}^{\downarrow}$ are determined as the highest energy at which $\kappa(E)$ crosses zero in the valence band for the majority and minority spins, respectively. By this method, we obtain for $d=2.4 \AA$ the values $\Phi_{\mathrm{CB}}^{\uparrow}=1.0$, $\Phi_{\mathrm{CB}}^{\downarrow}=1.6, \Phi_{\mathrm{VB}}^{\uparrow}=0.5$, and $\Phi_{\mathrm{VB}}^{\downarrow}=2.6 \mathrm{eV}$. For $d=2.8 \AA$, we obtain $\Phi_{\mathrm{CB}}^{\uparrow}=1.6, \Phi_{\mathrm{CB}}^{\downarrow}=2.2, \Phi_{\mathrm{VB}}^{\uparrow}=0.1$, and $\Phi_{\mathrm{VB}}^{\downarrow}=2.4 \mathrm{eV}$. The exchange splitting for the conduction band of $0.6 \mathrm{eV}$ matches the value for bulk EuO.

For the low-bias conductance, the thickness dependence of the transmission coefficient at $E_{F}$ is evaluated and from it the decay coefficient. In Fig. 12, we show $T\left(E_{F}\right)$ at $d=2.4 \AA$ and $d=2.8 \AA$ as functions of the EuO thickness. The decay rates correspond to those obtained in Fig. 11 at $E_{F}$. In Fig. 12(c), the effective spin filtering at $E_{F}$ is addressed. It can be seen that it increases towards $100 \%$ as $t$ increases.

\section{THE $I-V$ CURVE OF THE EUO SPIN FILTER}

The spin-resolved current-voltage $I-V$ characteristic and the spin polarization as functions of the bias voltage are shown in Fig. 13 for seven, nine, and $11 \mathrm{MLs}$ of EuO, and for both $d=2.4$ and $2.8 \AA$. The majority-spin current is higher than the minority-spin current for all bias voltages and for all 

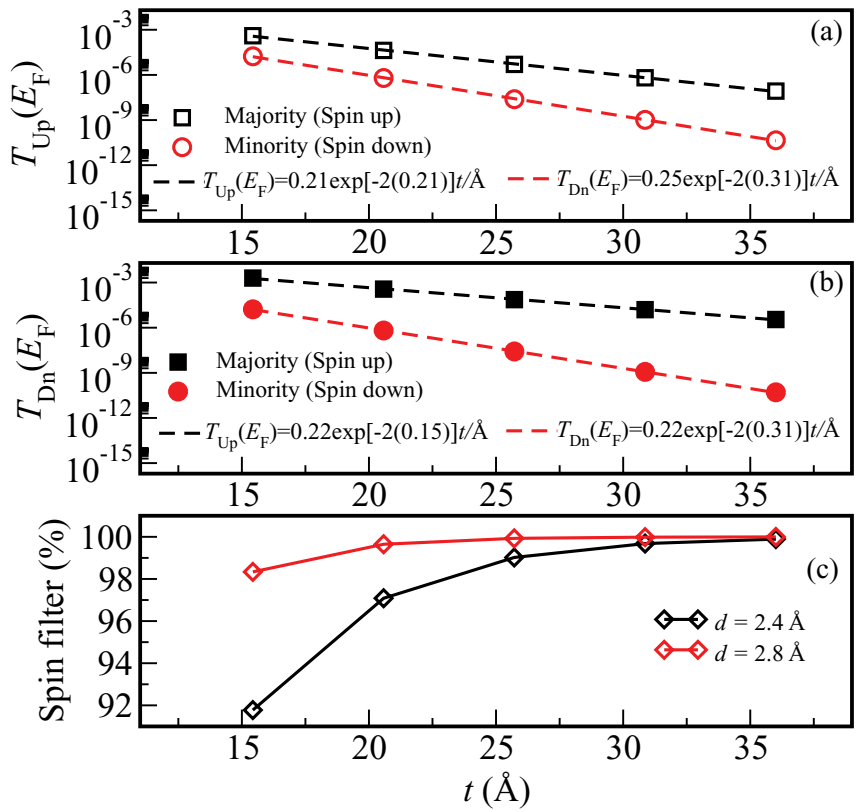

FIG. 12. (Color online) Dependence of $T\left(E_{F}\right)$ on $t$ for (a) $d=$ $2.4 \AA$ and (b) $d=2.8 \AA$. (c) The spin-filtering efficiency at $E_{F}$ for both values of $d$.

thicknesses, leading to large polarization in all cases. At low bias, there is a rapid increase of the tunneling current with the bias. This is typical for such systems due to the fact that the current is not only determined by electrons at $E_{F}$ but also by those in the energy range $E_{F} \pm e V / 2$ (bias window), for
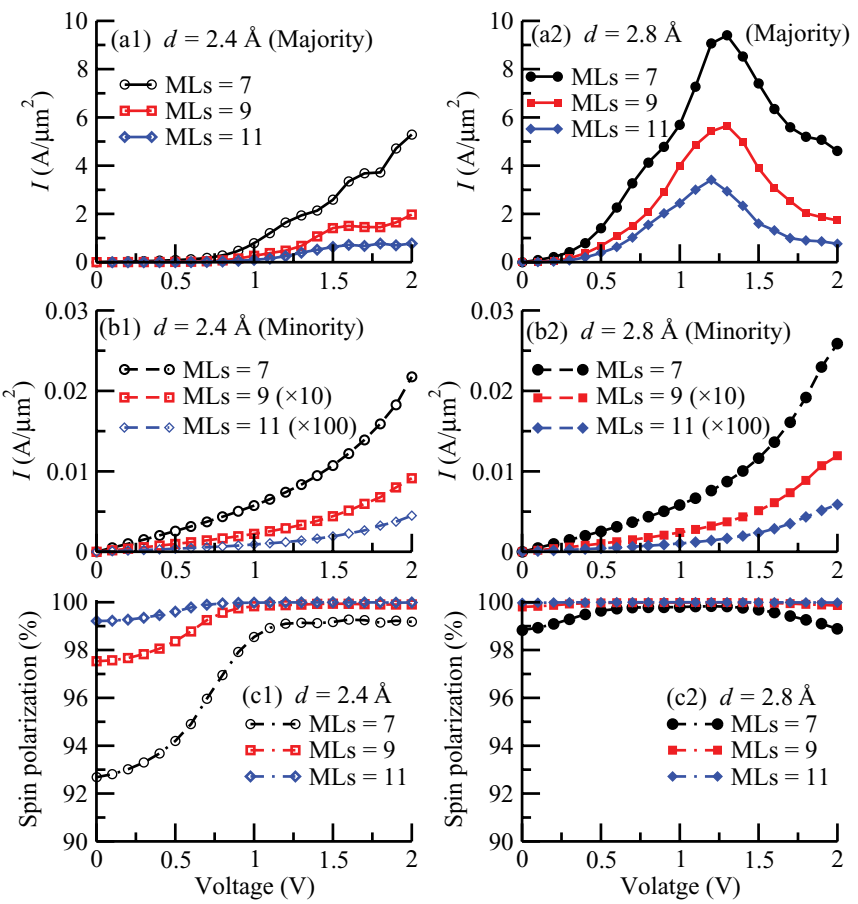

FIG. 13. (Color online) Spin-polarized current and spin polarization as functions of the bias voltage for different EuO thicknesses. (a1) Majority-spin current at $d=2.4 \AA$, (a2) majority-spin current at $d=2.8 \AA$, (b1) minority-spin current at $d=2.4 \AA$, (b2) minorityspin current at $d=2.8 \AA$, (c1) spin polarization at $d=2.4 \AA$, and (c2) spin polarization at $d=2.8 \AA$.
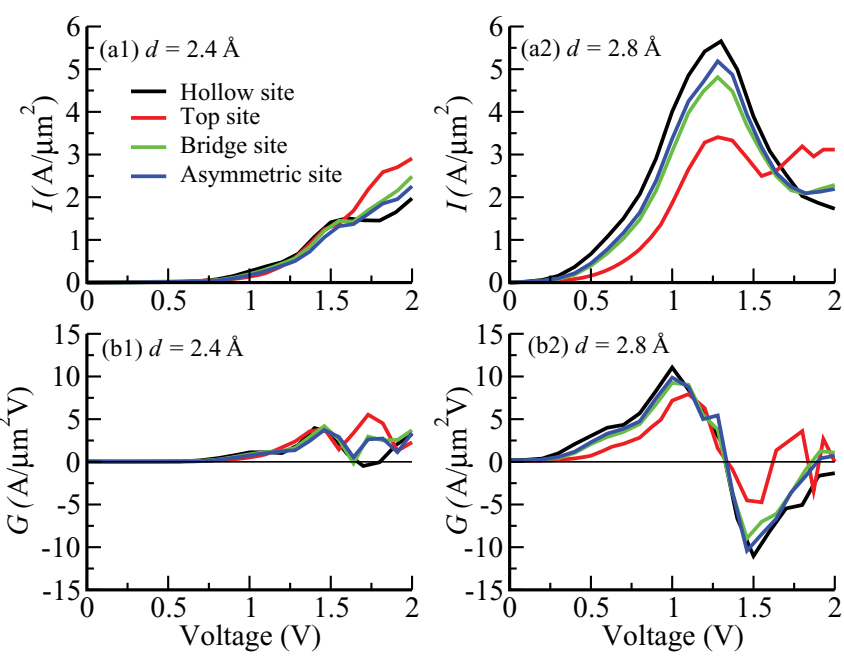

FIG. 14. (Color online) Total current and differential conductance for a $\mathrm{Cu} / \mathrm{EuO} / \mathrm{Cu}$ junction with a nine EuO MLs barrier at $d=2.4$ and $2.8 \AA$ with different interface geometries. (a1) Current at $d=$ $2.4 \AA$, (a2) current at $d=2.8 \AA$, (b1) conductance at $2.4 \AA$, and (b2) conductance at $2.8 \AA$.

which the barrier height can be reduced up to about $e V / 2$. At a bias of about $0.7 \mathrm{~V}$ for $d=2.4 \AA$, the electrons start to flow through the valence-band top, so that there is a sharp increase in current. The $I-V$ curve for $d=2.4 \AA$ is in good agreement with experimental results, ${ }^{22}$ showing a current onset at a bias of about $1 \mathrm{~V}$, and an increase in current at larger bias. Since for $d=2.8 \AA$ the valence band is very close to $E_{F}$, the onset is found at very low bias. The current increases up to about $1.3 \mathrm{~V}$, above which a decrease with increasing bias is found. This is caused by a reduction of the transmission over the entire bias window once the bias is very large. Such a current reduction results from the enhanced scattering as the potential is tilted inside the barrier due to the applied bias voltage. In these large scattering conditions, incoherent contributions to the current (not included here) are expected to play an important role.

For the minority spins, in contrast, the current remains in the tunneling regime for all bias voltages due to the large barrier height. The large difference between majority- and minority-spin currents is reflected by a high spin polarization $P$ defined as $\left(I^{\uparrow}-I^{\downarrow}\right) /\left(I^{\uparrow}+I^{\downarrow}\right)$ [see Fig. 13(c1)]. $P$ remains large for all the bias voltages considered due to the small $I^{\downarrow}$. We note that the spin splitting of the conduction band plays only a secondary role in our results, since the majority current is mainly determined by the valence-band contribution (which is not sensitive to the exact position of $E_{F}$ inside the $\mathrm{EuO}$ energy gap). For $n$-type $\mathrm{EuO}$, one expects a pinning of the conduction band to $E_{F}$, leading to an almost metalliclike character dominated by the $\mathrm{EuO}$ conduction electrons.

The dependences of the $I-V$ characteristics and of the differential conductance $G(V)=d I / d V$ on the $\mathrm{Cu} / \mathrm{EuO}$ interface geometry are addressed in Fig. 14. Overall, the $I$ - $V$ characteristics are similar for all the interfaces, which reflects the fact that the zero-bias transmission is also rather similar (see Fig. 9). For $d=2.4 \AA$, there is a sharp increase in $G$ at the current onset, whereas it then stays approximately constant. For $d=2.8 \AA$, in contrast, $G$ is large at low bias and changes sign at $1.3 \mathrm{~V}$. 


\section{CONCLUSIONS}

We have studied the electronic structure, the complex band structure, and the spin transport properties of epitaxial $\mathrm{Cu} / \mathrm{EuO} / \mathrm{Cu}$ tunnel junctions. The spin transport properties of EuO epitaxially grown on $\mathrm{Cu}$ are dominated by the Eu-4 $f$ valence states and $\mathrm{Eu}-5 d$ conduction states. We show that EuO acts as an almost perfect spin filter, where close to $100 \%$ spin polarization can be achieved. The polarization increases with increasing EuO thickness as expected from the complex band structure, where the decay of the wave function into $\mathrm{EuO}$ is predicted to be much smaller for the majority spins than for the minority ones. Since the conduction states of EuO have no $\Delta_{1}$ symmetry to match the states in the $\mathrm{Cu}$ electrodes, the gap in the transmission coefficient is significantly larger than the band gap of EuO. Under a bias voltage, the spin polarization of the current does not decrease, as in usual tunnel junctions, but remains approximately constant up to all considered bias voltages.

\section{ACKNOWLEDGMENTS}

We would like to acknowledge technical assistance from Apirat Siritarathiwat and Hao Wang. N.J. and U.E. acknowledge financial support by the Deutsche Forschungsgemeinschaft through TRR 80. Computational resources have been provided by TCHPC Ireland and LRZ Munich Germany. I.R. and S.S. acknowledge financial support by KAUST (ACRAB project).
${ }^{1}$ S. Yuasa and D. D. Djayaprawira, J. Phys. D: Appl. Phys. 40, R337 (2007).

${ }^{2}$ S. X. Huang, T. Y. Chen, and C. L. Chien, Appl. Phys. Lett. 92, 242509 (2008).

${ }^{3}$ J. S. Moodera, T. S. Santos, and T. Nagahama, J. Phys.: Condens. Matter 19, 165202 (2007).

${ }^{4}$ W. H. Butler, X.-G. Zhang, T. C. Schulthess, and J. M. MacLaren, Phys. Rev. B 63, 054416 (2001).

${ }^{5}$ J. Mathon and A. Umerski, Phys. Rev. B 63, 220403(R) (2001).

${ }^{6}$ I. Rungger, O. Mryasov, and S. Sanvito, Phys. Rev. B 79, 094414 (2009).

${ }^{7}$ C. Heiliger, P. Zahn, and I. Mertig, Mater. Today 9, 46 (2006).

${ }^{8}$ H. Itoh, J. Phys. D: Appl. Phys. 40, 1228 (2007).

${ }^{9}$ C. Tiusan, M. Hehn, F. Montaigne, F. Greullet, S. Andrieu, and A. Schuhl, J. Phys.: Condens. Matter 19, 165201 (2007).

${ }^{10}$ W. H. Butler, Sci. Technol. Adv. Mater. 9, 014106 (2008).

${ }^{11}$ G. X. Miao, M. Müller, and J. S. Moodera, Phys. Rev. Lett. 102, 076601 (2009).

${ }^{12}$ G. X. Miao, M. Münzenberg, and J. S. Moodera, Rep. Prog. Phys. 74, 036501 (2011).

${ }^{13}$ M. Julliere, Phys. Lett. A 54, 225 (1975)

${ }^{14}$ A. Mauger and C. Godart, Phys. Rep. 141, 51 (1986).

${ }^{15}$ P. G. Steeneken, L. H. Tjeng, I. Elfimov, G. A. Sawatzky, G. Ghiringhelli, N. B. Brookes, and D.-J. Huang, Phys. Rev. Lett. 88, 047201 (2002).

${ }^{16}$ T. S. Santos and J. S. Moodera, Phys. Rev. B 69, 241203(R) (2004).

${ }^{17}$ E. Negusse, J. Holroyd, M. Liberati, J. Dvorak, Y. U. Idzerda, T. S. Santos, J. S. Moodera, and E. Arenholz, J. Appl. Phys. 99, 08E507 (2006).

${ }^{18}$ T. S. Santos, J. S. Moodera, K. V. Raman, E. Negusse, J. Holroyd, J. Dvorak, M. Liberati, Y. U. Idzerda, and E. Arenholz, Phys. Rev. Lett. 101, 147201 (2008).

${ }^{19}$ S. M. Watson, T. S. Santos, J. A. Borchers, and J. S. Moodera, J. Appl. Phys. 103, 07A719 (2008).

${ }^{20}$ E. Negusse, J. Dvorak, J. S. Holroyd, M. Liberati, T. Santos, J. Moodera, E. Arenholz, and Y. U. Idzerda, J. Appl. Phys. 105, 07C930 (2009).

${ }^{21}$ M. Müller, G. X. Miao, and J. S. Moodera, J. Appl. Phys. 105, 07C917 (2009)

${ }^{22}$ M. Müller, G. X. Miao, and J. S. Moodera, Europhys. Lett. 88, 47006 (2009).
${ }^{23}$ J. M. Soler, E. Artacho, J. D. Gale, A. García, J. Junquera, P. Ordejon, and D. Sańchez-Portal, J. Phys.: Condens. Matter 14, 2745 (2002).

${ }^{24}$ V. I. Anisimov, J. Zaanen, and O. K. Andersen, Phys. Rev. B 44, 943 (1991).

${ }^{25}$ V. I. Anisimov, I. V. Solovyev, M. A. Korotin, M. T. Czyzyk, and G. A. Sawatzky, Phys. Rev. B 48, 16929 (1993).

${ }^{26}$ N. J. C. Ingle and I. S. Elfimov, Phys. Rev. B 77, 121202(R) (2008).

${ }^{27}$ A. R. Rocha, V. M. García-Suárez, S. Bailey, C. Lambert, J. Ferrer, and S. Sanvito, Nature Mater. 4, 335 (2005).

${ }^{28}$ A. R. Rocha, V. M. García-Suárez, S. Bailey, C. Lambert, J. Ferrer, and S. Sanvito, Phys. Rev. B 73, 085414 (2006).

${ }^{29}$ I. Rungger and S. Sanvito, Phys. Rev. B 78, 035407 (2008).

${ }^{30}$ D. Chen, X. L. Ma, and Y. M. Wang, Phys. Rev. B 75, 125409 (2007).

${ }^{31}$ M. M. Fadlallah, C. Schuster, U. Schwingenschlögl, I. Rungger, and U. Eckern, Phys. Rev. B 80, 235332 (2009).

${ }^{32}$ D. B. Ghosh, M. De, and S. K. De, Phys. Rev. B 70, 115211 (2004).

${ }^{33}$ J. Kunes, W. Kuy, and W. E. Pickett, J. Phys. Soc. Jpn. 74, 1408 (2005).

${ }^{34}$ H. Miyazaki, T. Ito, S. Ota, H. J. Im, S. Yagi, M. Kato, K. Soda, and S.-I. Kimura, Phys. B 403, 917 (2008).

${ }^{35}$ E. Bousquet, N. A. Spaldin, and P. Ghosez, Phys. Rev. Lett. 104, 037601 (2010).

${ }^{36}$ J. M. An, S. V. Barabash, V. Ozolins, M. van Schilfgaarde, and K. D. Belashchenko, Phys. Rev. B 83, 064105 (2011).

${ }^{37}$ H. Miyazaki, T. Ito, H. J. Im, K. Terashima, S. Yagi, M. Kato, K. Soda, and S. Kimura, Jpn. J. Appl. Phys. 48, 055504 (2009).

${ }^{38}$ H. Miyazaki, T. Ito, H. J. Im, S. Yagi, M. Kato, K. Soda, and S. Kimura, Phys. Rev. Lett. 102, 227203 (2009).

${ }^{39}$ N. M. Caffrey, T. Archer, I. Rungger, and S. Sanvito, Phys. Rev. B 83, 125409 (2011).

${ }^{40}$ C. Toher, I. Rungger, and S. Sanvito, Phys. Rev. B 79, 205427 (2009).

${ }^{41}$ I. Rungger, X. Chen, U. Schwingenschlögl, and S. Sanvito, Phys. Rev. B 81, 235407 (2010).

${ }^{42}$ F. Favot, A. Dal Corso, and A. Baldereschi, J. Chem. Phys. 114, 483 (2001). 
${ }^{43}$ P. O. Gartland, S. Berge, and B. J. Slagsvold, Phys. Rev. Lett. 28, 738 (1972).

${ }^{44}$ M. Weinelt, J. Phys.: Condens. Matter 14, R1099 (2002).

${ }^{45}$ D. E. Eastman, Phys. Rev. B 8, 6027 (1973).

${ }^{46}$ M. Barbagallo, N. D. M. Hine, J. F. K. Cooper, N.-J. Steinke, A. Ionescu, C. H. W. Barnes, C. J. Kinane, R. M. Dalgliesh, T. R. Charlton, and S. Langridge, Phys. Rev. B 81, 235216 (2010)
${ }^{47}$ X. Wang, P. Liu, K. A. Fox, J. Tang, J. A. Colón Santana, K. Belashchenko, P. A. Dowben, and Y. Sui, IEEE Trans. Magn. 46, 1879 (2010).

${ }^{48}$ J. A. Colon Santana, J. M. An, N. Wu, K. D. Belashchenko, X. Wang, P. Liu, J. Tang, Y. Losovyj, I. N. Yakovkin, and P. A. Dowben, Phys. Rev. B 85, 014406 (2012).

${ }^{49}$ X. Shen, L. Sun, E. Benassi, Z. Shen, X. Zhao, S. Sanvito, and S. Hou, J. Chem. Phys. 132, 054703 (2010). 